\title{
BIOMECHANICAL ROOT CAUSE ANALYSIS OF COMPLICATIONS IN HEAD IMMOBILIZATION DEVICES FOR PEDIATRIC NEUROSURGERY
}

\author{
Moataz Abdulhafez \\ Department of Industrial Engineering \\ University of Pittsburgh \\ Pittsburgh, PA, USA \\ Mohamed Zaazoue \\ Department of Neurosurgery \\ Boston Children's Hospital \\ Indiana University School of Medicine \\ Department of Neurosurgery \\ Indianapolis, IN, USA
}

\author{
Karim Kadry \\ Department of Industrial Engineering \\ University of Pittsburgh \\ Pittsburgh, PA, USA \\ American University in Cairo \\ Department of Mechanical Engineering
}

\author{
Liliana C. Goumnerova \\ Department of Neurosurgery \\ Boston Children's Hospital \\ Harvard Medical School \\ Boston, MA, USA
}

\author{
Mostafa Bedewy* \\ Department of Industrial Engineering \\ University of Pittsburgh \\ Pittsburgh, PA, USA \\ *mbedewy@pitt.edu
}

\section{KEYWORDS}

Head immobilization devices, finite element modeling, agedependent bone properties, pediatric, bone

\begin{abstract}
Precise and firm fixation of the cranium is critical during craniotomy and delicate brain neurosurgery making head immobilization devices (HIDs) a staple instrument in brain neurosurgical operations today. However, despite their popularity, there is no standard procedure for their use and many complications arise from using HIDs in pediatric neurosurgery. In this paper, we identify biomechanical causes of complications and quantify risks in pin-type HIDs including clamping force selection, positioning and age effects. Based on our root cause analysis, we develop a framework to address the biomechanical factors that influence complications and understand the biomechanics of the clamping process. We develop an agedependent finite element model (FEM) of a single pin on a cranial bone disc with the representative properties and skull
\end{abstract}

thickness depending on age. This model can be utilized to reduce risk of complications by design as well as to provide recommendations for current practices.

\section{INTRODUCTION}

Head immobilization devices (HIDs) are among the most commonly used surgical tools in neurosurgery, otolaryngology and orthopedic procedures. These simple devices serve two main functions: (1) to support the weight of the patient's head, and (2) to immobilize the head during surgical manipulation. Several commercially available HIDs exist, all of which share some common features. The vast majority of available HIDs are pintype devices that depend on pins applied to the skull to achieve stabilization. These devices also contain a torque screw or similar mechanism integrated with one or more of the pins that is tightened by hand during clamping to achieve robust immobilization as shown in Figure 1.

Several types of HID-related complications have been reported in literature, for both the adult and pediatric

This is the author's manuscript of the article published in final edited form as:

Abdulhafez, M., Kadry, K., Zaazoue, M., Goumnerova, L. C., \& Bedewy, M. (2018, June 18). Biomechanical Root Cause Analysis of Complications in Head Immobilization Devices for Pediatric Neurosurgery. V001T05A007-V001T05A007. 
populations. These complications include depressed skull fractures, epidural hemorrhage, tension pneumocephalus [1], and venous air embolism [2]. Importantly, pediatric patients are more likely to suffer from such complications owing to their skull's lower mechanical strength and smaller thickness of their skull bones [2-5].

A clinical survey for pediatric neurosurgeons conducted by Berry et al. [5] in 2008 showed that 89 out of 142 respondents (54\%) experienced complications related to HIDs. However, the actual published pediatric complications in the literature are much less than that, which clearly indicates that this is a common, yet under-reported problem, [2-4,6-11]. In our previous work in which we reviewed HID related complications in one institution, we found that these injuries occurred more with pediatric cases rather than adults, and identified other medical conditions such as hydrocephalus for cases we reviewed [12]. Recently, the U.S. Food and Drug Administration (FDA) has issued a safety communication,[13] stating that it has received more than 1,000 medical device reports (MDRs) associated with HID-related adverse events, during the period between January 2009 and January 2016, resulting in more than 700 injuries.

Unfortunately, there is a lack of clear guidelines related to clamping procedure for pediatric cases, Berry [5] found that $94 \%$ of the 156 pediatric neurosurgeons were not aware of published guidelines for the use of HIDs in children. This is reflected in the variability of clamping force for each age range in the same survey. This problem was also emphasized in our work [12], however, the lack of available data prevented a reliable set of guidelines to be created and enforced.

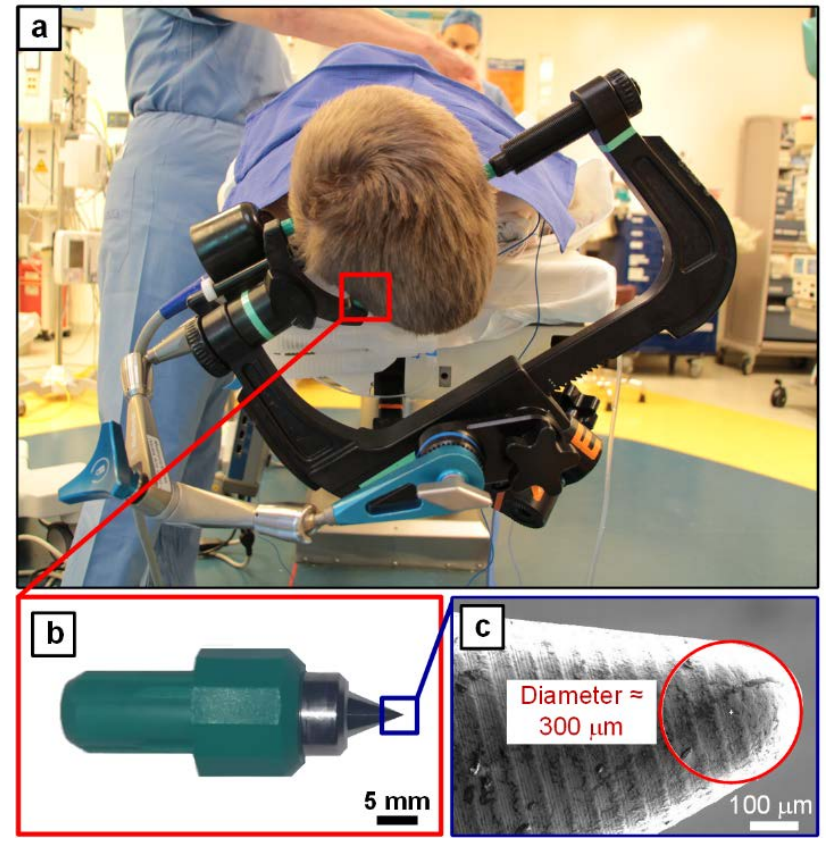

Figure 1: (a) HID device clamping a pediatric patient's head, (b) Image of pediatric type HID pin, (c) SEM image of pin tip
In this paper, we discuss the risk factors attributed to pin-type HIDs through a root cause analysis of possible complications. We focus on biomedical age-dependent risks, in which we propose a model of the evolution of parietal bone properties with age. Additionally, we present a finite element model (FEM) of the pin-bone interface that incorporates the age-dependent model. This model is proposed as a framework to assess the risk of excessive penetration, to understand the mechanics of bone penetration for different clamping forces. Hence, it is envisioned as a tool in the design of new pins and HID devices.

\section{CLASSIFICATION OF COMPLICATIONS' CAUSES}

In order to address the complications issues of pin type HID devices, we conduct a root cause analysis of the possible complications and we divide the possible causes of complications into three categories, as shown in Figure 2: (1) human error; (2) lack of patient-specific data, and (3) HID design (including pin and system design).

\section{Human error}

Complications attributed to human errors could be due to excessively high or low clamping forces, poor pin selection and placement; any of which, could eventually lead to complications like slipping of the head or excessive penetration and skull fracture.

\section{Lack of patient-specific data}

Complications could occur due to lack of information on the patient, which could indicate skull bone structure and properties, skull thickness, or any other relevant medical conditions. Additionally, the value of the applied clamping force/stress and penetration/strain during clamping is unknown as well, because the commercially available HIDs are not typically used to measure those values. While some of this data can currently be inferred from the patient's age and medical record or from the device, it is difficult to obtain qualitative data of the patient's skull strength and skull thickness before surgery. Also, after clamping the skull, it is difficult to measure the amount of achieved penetration into the skull or the resulting stresses.

\section{HID Design}

Design features (or lack thereof) could potentially contribute to complication risks or reduce the risk of complications due to human error or lack of patient data. For example, unequal force distribution of pin load among the multiple pins in a single HID device can lead to excessive loads to act locally on the skull. Another issue is the lack of alarms in the system in the case of dangerous loading conditions and the lack of built-in fail safe mechanisms. These issues can be mitigated by employing relevant mechanical design principles, such as including failsafes, alarms and sensors in the system design as well as improving the pin design or adding supports. Some of these concepts have been proposed in patents [14-16] and reports from literature [17], however few have reached the market, and many more have not been proposed. 


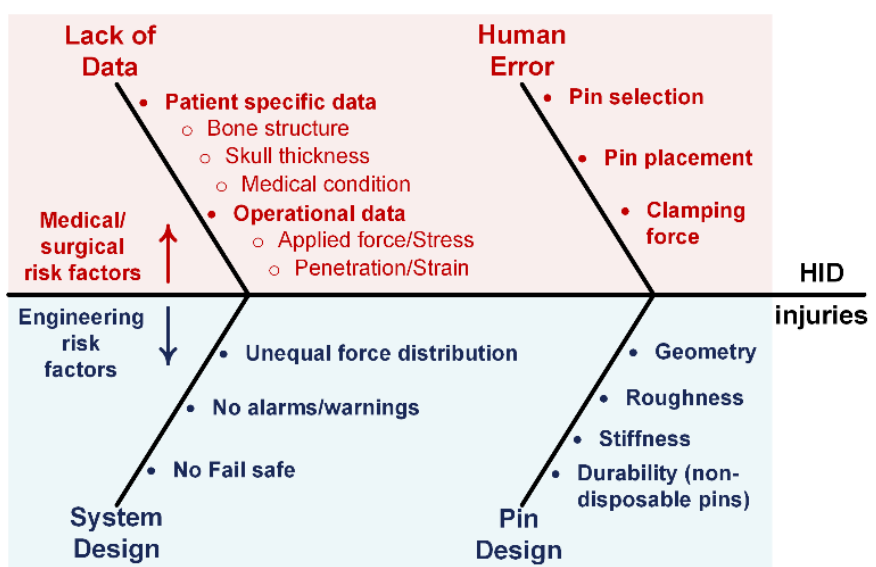

Figure 2: Fishbone (Ishikawa) diagram for causes of HID-related complications and injuries

\section{SELECTED BIOMECHANICAL FACTORS}

In this section, we emphasize and discuss some of the critical biomechanical risk factors presented. In general, the underlying biomechanics of pin bone interaction when tightening the pin and under loading after clamping are not fully understood, especially when considering the complex nature of skull bone tissue, age-dependent factors, patient-specific issues and the use of multiple pins. In an effort to provide a tool to understand this, we discuss the most important biomechanical factors attributing to complications, namely pin geometry, pin placement and clamping force selection by the user as well as the patient's age.

\section{Pin design}

Pin geometry plays an important role in how the pin interacts with the skull bone, where the cone angle and the pin tip radius of curvature correlate with the stresses forming in the skull bone and the subsequent deformation. For commercial pin type HIDs, manufacturers typically offer two types of pins: pediatric and adult models. They are offered in reusable or disposable variants (e.g. for MRI-compatible HIDs and non-MRI-compatible HIDs). The pediatric pins are characterized by a larger cone angle $(\theta)$, when compared to the adult model as shown in Figure 3(a,b). Other types of pins, namely pins for halo-vests, which are not typically used in neurosurgery, exhibit a curved profile, in contrast to the linear profile of HID type pins to control amount of pin penetration vs. the amount of force applied [18] as shown in Figure 3(c).

\section{Clamping force}

The clamping force applied by the user is a critical parameter that influences the amount of pin penetration into the skull. Excessive penetration could lead to dural puncture, while insufficient penetration could lead to slippage of the head. Manufacturers broadly recommend a force of $60 \mathrm{lbs}(267 \mathrm{~N})$ for adults and $40 \mathrm{lbs}(178 \mathrm{~N})$ for children and typically warn against exceeding 80 lbs (356 N) of force. Despite its importance, there is no standard way to decide the clamping force, owing to the difference in conditions and skull thickness for each patient.

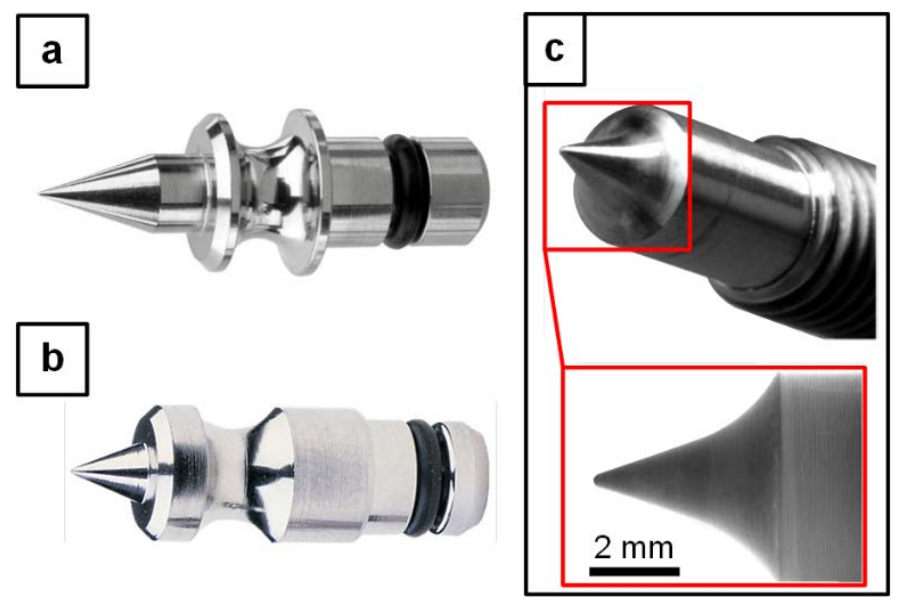

Figure 3: Different types of skull penetrating pins: (a) Adult MAYFIELD ${ }^{\circledR}$ type reusable skull pins, (b) Pediatric MAYFIELD $^{\circledR}$ type reusable skull pins [19], (c) Halo vest type pin with inset showing SEM of pin tip (Adapted from reference [20])

\section{Pin Placement}

Pin placement and device setup is an important factor that also influences the risk of complication. Typically, Manufacturers provide general guidelines for pin placement, under no liability [21].

In practice, the surgeon clamping the patient's skull decides the force, location and orientation of the frame and the pin engagement points on the skull based on the location of the surgery and the manufacturer recommendations, yet complications can occur due to sub-optimal orientation and location, as well as over-tightening and patient specific conditions. In order to achieve a stationary and stabilized skull, HIDs need to both immobilize the skull and carry its weight.

The forces acting on a body in static equilibrium balance each other. Hence, the pin reaction forces have to balance the downward force of the skull's own weight. As shown in Figure 4, for the same head orientation, different HID fixation positions would lead to different reaction forces on the pins. Small modifications in the orientation of the HID as shown in Figure 4(B, D) by repositioning the 2-pin side at a lower level, increases the vertical components of the forces exerted to react to the downward weight. This lowers the forces and pressures at the points of contact and thus reduce fracture risks. Practically, a compromise from optimal HID positioning would be needed to avoid obstructions to the surgical field by the pins.

\section{Age dependence}

Patient age is also a critical factor that affects complication risks. Adult skull bone is generally composed of three layers: an outer cortical layer and inner cortical layer sandwiching a trabecular layer as shown in the figure. Cortical bone is characterized by being more dense and compact, while trabecular bone has a cellular structure with trabeculae forming the basic strut elements. At birth, the skull bone is mostly composed of a single cortical layer [23]. With age, trabecular tissue develops in the 
center of the cortical tissue, forming the three layer structure. Additionally, the mechanical properties of the bone tissue evolves with age as well as shown in figure. Both types of bone tissue exhibit different mechanical properties that are generally dependent on direction, age, location and medical condition.

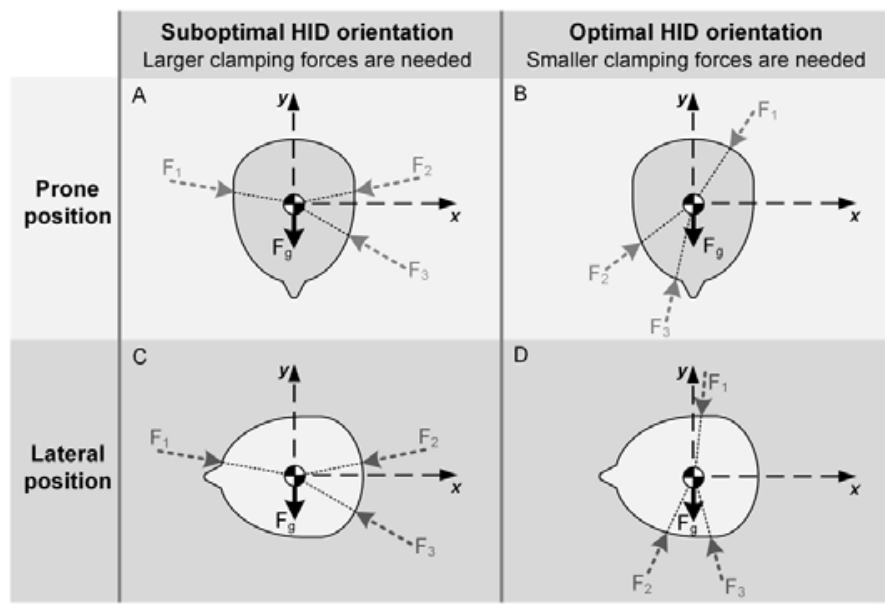

Figure 4: Schematic of free body diagrams for a clamped skull using a HID for two different surgical head orientations: Prone $(A, B)$ and lateral (C,D). For each position, two different orientation are shown, where the resultant forces for each case is shown where an optimal orientation (better force orientation) is demonstrated.

\section{FINITE ELEMENT MODELING}

As seen in the previous section, in practice surgeons depend on rules of thumb to decide the clamping conditions, as there are no standard procedures to clamping condition selection. This motivates the development of tools that aid clamp users and surgeons in the decision making process. In what follows, we propose a FEM model that can assist in explaining the underlying biomechanics of the pin penetration process and can be used as a tool for clamping force selection and head orientation given the age and health condition of the patient.

\section{FEM model}

The basic geometry of the FEM is shown in the Figure 5(a); it consists of a pin positioned above a curved disc divided into three layers with radius of curvature $(R)$. Half symmetry is exploited due to problem symmetry. The top layer denotes the outer cortical table with thickness $\left(\mathrm{t}_{\mathrm{co}}\right)$, the middle denotes the trabecular layer with thickness $\left(t_{d}\right)$ and the bottom layer denotes the inner cortical table with thickness $\left(\mathrm{t}_{\mathrm{ci}}\right)$. The disc end surfaces are constrained from moving, while the cylindrical surfaces of the pin are constrained against rotation.

The geometry and material properties of the bone disc are assigned according the age dependent model described in the next section. A pediatric type pin is used in the simulation. The simulation is divided into two load steps as shown in Figure 5(c). The first load step is an axial load applied on the top of the pin, corresponding to the initial pin tightening process. We select loads corresponding to 20 to $80 \mathrm{lbs}$ in $20 \mathrm{lb}$ increments, the operational range of HID pins. In the second load step, a lateral load is applied to the top face of the pin to simulate lateral loading on the pin during intraoperative maneuvers or due to skull weight. The lateral load magnitude is set to approximately equal to the appropriate head weight. The weight of the head was chosen as the lateral force to correspond to a worst case loading scenario. In reality the lateral force on a single pin will be less than the weight of the head, this is due to two factors, the first is that the headrest will generally take most of the weight of the head, leaving little weight to be carried by the pins, the second reason is that due to the multiple pin arrangement, the weight of the head is distributed among multiple pins.
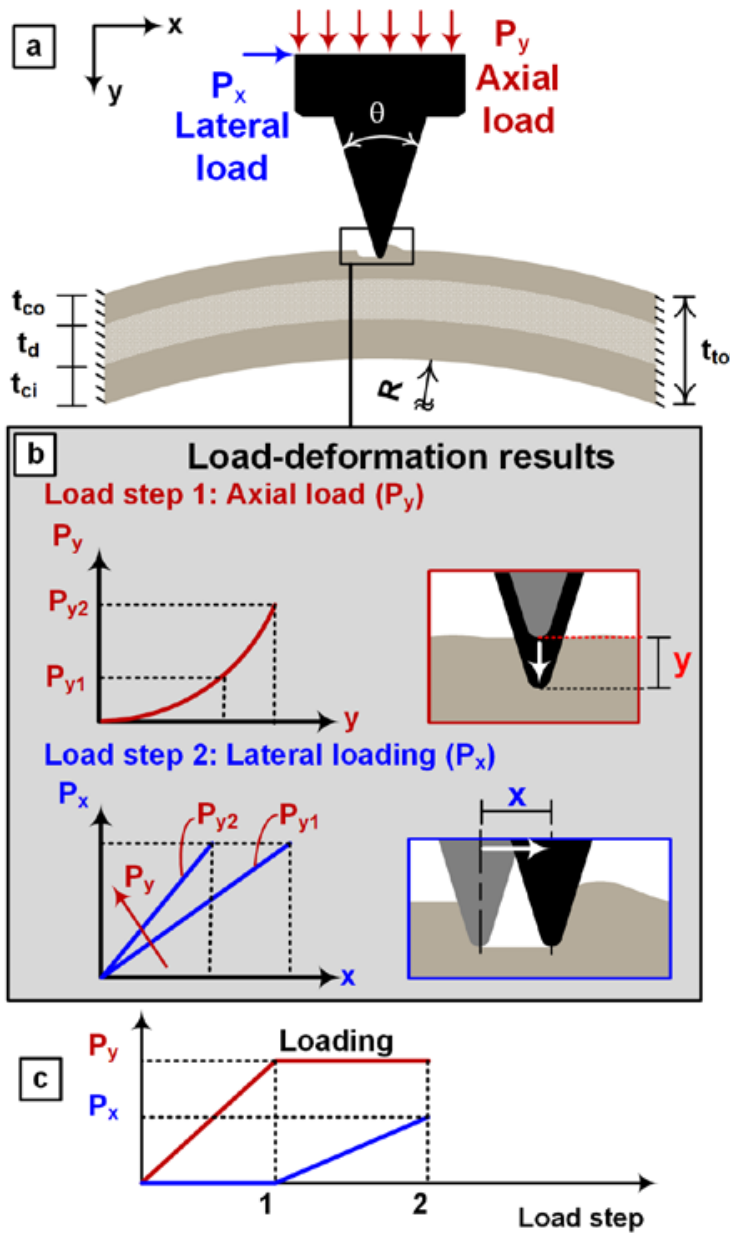

Figure 5: Finite Element Problem (a) definition and boundary conditions, (b) results in the axial and lateral directions and (c) the load stepping used in the simulations

The output of the simulation is the load-displacement curve resulting from the tightening process and the lateral stiffness at different axial loads as shown in Figure 5(b). As a measure of skull failure/excessive penetration, we set a criterion of the maximum lateral penetration being larger than $90 \%$ of the upper cortical layer. This criterion is usually assumed for halo type pin analysis [22]. This is based on the expectation that failure 
constitutes complete pin penetration of the top cortical layer. Due to relative weakness of the trabecular layer compared to the cortical layers, we believe this is a valid criterion.

The model is composed of 3D tetrahedral elements with the elements in the upper cortical layer and the pin type refined. Frictional contact is assumed between the pin tip and upper cortical layer with 0.2 as the frictional coefficient. Adaptive remeshing is activated during the simulation, to re-mesh any highly skewed elements that were distorted during the simulation in the upper cortical layer. The model is run using ANSYS 18.1 with a sample mesh and pin geometry shown in Figure 6. The dimensions of the pediatric pin used are $(\mathrm{d}=7.5 \mathrm{~mm}, \mathrm{q}=4 \mathrm{~mm}$, $\mathrm{l}=5 \mathrm{~mm}, \mathrm{r}=0.15 \mathrm{~mm}$ ).

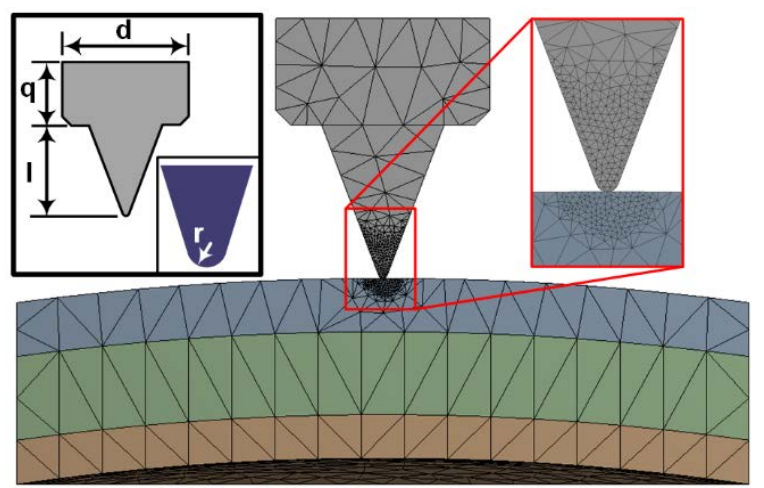

Figure 6: Schematic of pin geometry and example of the used mesh for the problem

\section{Age dependent model for skull bone properties}

To address and understand the biomechanical factors presented, we start by developing a qualitative model charting the change of properties with age using data from literature covering pediatric patients up to adulthood. This model will be integrated into the finite element model presented. With age as input, the age dependent model of the properties should output the average thicknesses of the different layers, as well as the ultimate strength and Young's modulus of the different tissue in the principal directions as schematically shown in Figure 7.

In order to determine the geometric parameters of the skull for each age, the relationship between total thickness of the cranial wall with age has to be modelled. Furthermore, the evolution of the individual cortical and trabecular thicknesses must be determined as well. To achieve this, a survey on the current literature was conducted in order to construct a growth model for the total thickness of the skull for the lifetime of a human being. Data was collated without regard to the difference between sex or race. Since the properties change with bone type and location, we select data that is localized to the parietal bone of the skull. The curvature of the bone layer is also included. All data was taken from skulls that had no bone affecting conditions.
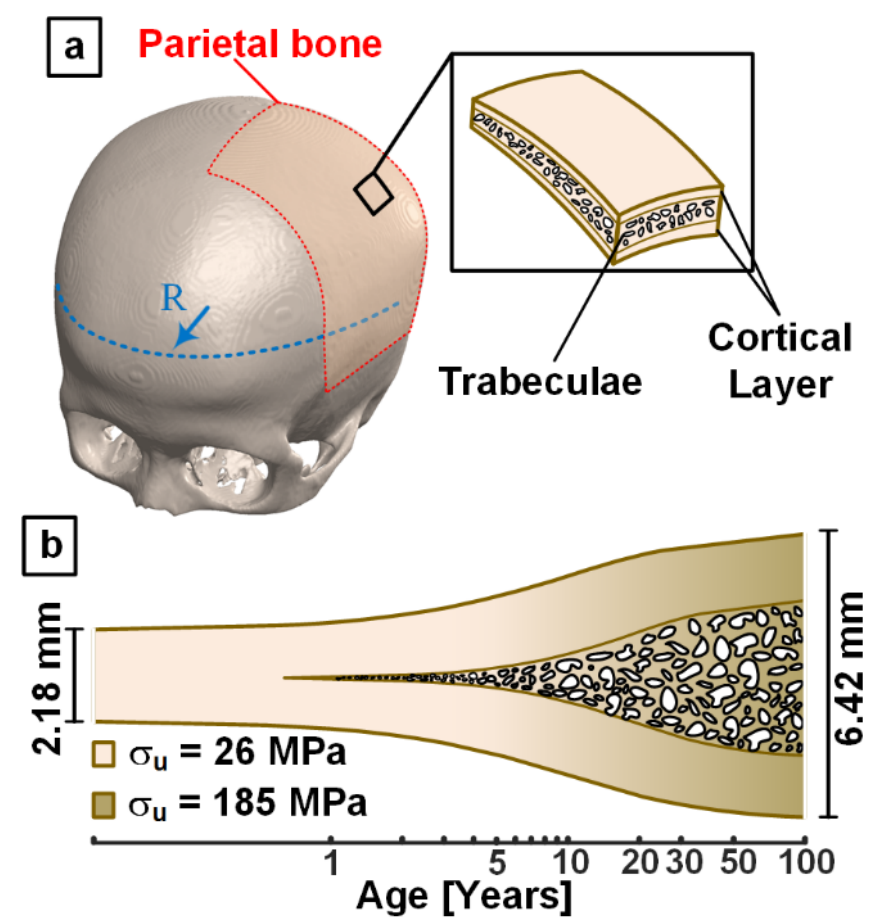

Figure 7: (a) Schematic illustrating the location of parietal bone and skull radius and morphology of skull bone, (b) Schematic illustrating the age dependent model for average parietal bone skull thickness and mechanical properties

The data utilized is collected from studies by Loder [23], Kriewall [24], Li [25], Garfin [26], Wong [27], Desouza [28], Moreira [29], Sullivan [30], Voie [31] and Lillie [32] on adult and pediatric parietal bone structure and thickness as shown in Figure 8(a) In order to include data regarding gestational period skull thicknesses, the point of birth was considered to be 9 months after conception, with a post conception age of 36 weeks corresponding to the 0 point on the $\mathrm{X}$-axis. Furthermore, whenever the thickness was given in tandem with an age range and sample size, an average age between the extremes was assumed, with the number of identical points corresponding to the given sample size.

A monomolecular model of the form

$$
y=A\left(1-e^{-k\left(x-x_{c}\right)}\right)
$$

was used to mathematically fit the evolution of total cranial thickness with age, since data shows rapid growth during the birth-maturity period, with a stabilization in thickness after adulthood is reached. In the equation, $\mathrm{y}$ is the skull thickness corresponding to age $\mathrm{x}, \mathrm{A}$ is the maximum curve value, $\mathrm{k}$ is the curve steepness and $x_{c}$ is the age corresponding to the curves midpoint. It has been shown that the aforementioned monomolecular model has been used previously to model bone growth of animals in previous studies conducted by Lee [33],Cooper [34], Erickson [35] and Høye [36]. The fit had an $\mathrm{R}^{2}$ value of 0.95 , suggesting that the assumption is valid. By using this model, the total skull thickness at birth is estimated to be 
$2.183 \mathrm{~mm}$ while the thickness asymptotically approaches 6.4 $\mathrm{mm}$ with old age as seen in Figure 8(a)
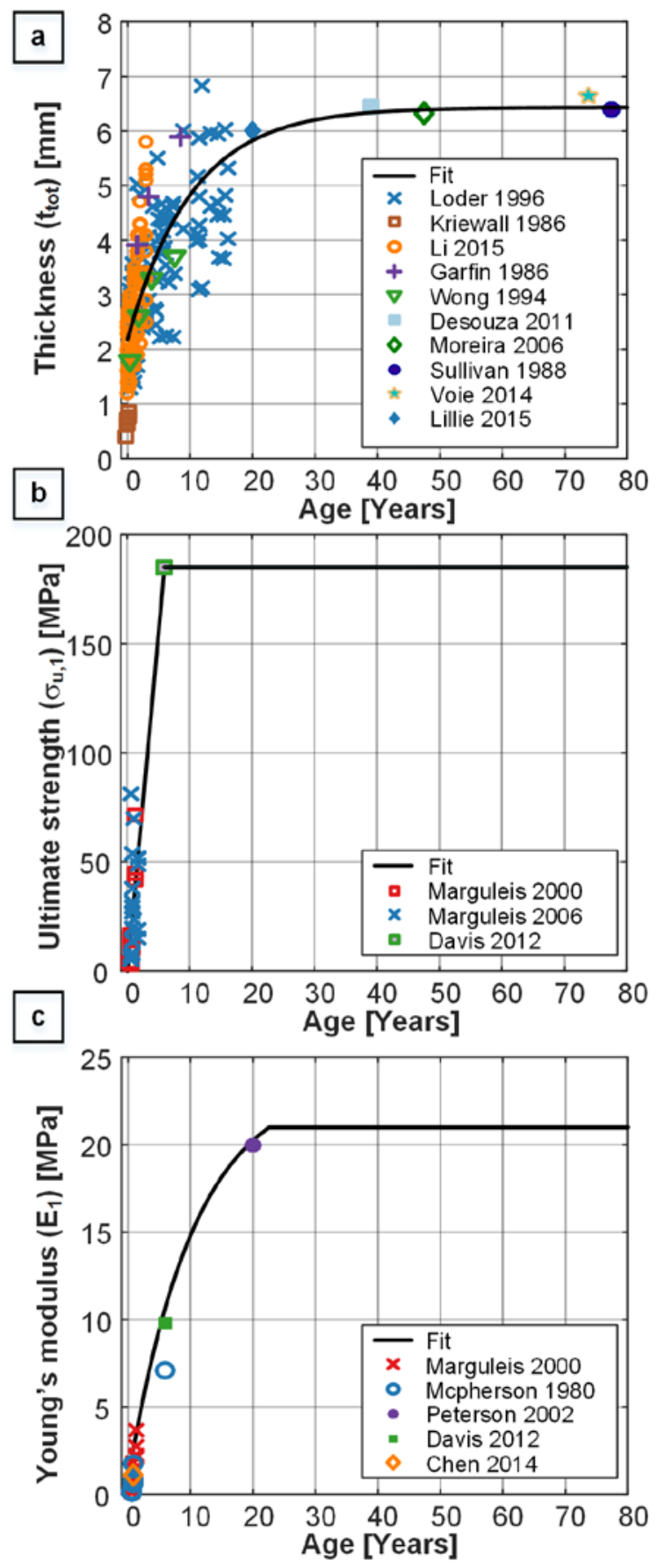

Figure 8: Evolution of (a) total parietal cranial bone thickness, (b) ultimate strength, (c) Young's modulus of cortical parietal bone with age, collected from different sources in literature

Data from Huang [37] was used to find the ratio between trabeculae thickness and total thickness. The thickness scatterplot for the trabeculae layer was used and monomolecular fit was established for parietal trabecular thickness data which included ages above 3 years. The same was done for the parietal total thickness. Once both equations were found, they were divided by each other in order to produce an equation detailing the ratio between trabecular thickness and total thickness with age. The ratio between the trabecular and total thicknesses was obtained by dividing the linear equation of the trabecular thickness by the linear equation of the total thickness. Furthermore, the ratio between the outer cortical and inner cortical table was assumed to be constant and approximately equal to 0.8147 by averaging the inner-outer cortical ratio between males and females for the 20 year old parietal bone data found in Lillie [32]. It is with these parameters that the individual thicknesses can be estimated from the total calculated thickness at a certain age.

\section{Material model}

Both cortical and trabecular bone tissue exhibit anisotropic material properties [38].The cortical layers were assumed to be transversely isotropic. For adults, the stiffness parameters of the outer cortical layer taken from ultrasonic studies conducted by Peterson [39] on human parietal bone. In order to obtain transversely isotropic parameters, averages were taken between certain material parameters such as $\mathrm{E}_{\mathrm{X}}$ and $\mathrm{E}_{\mathrm{z}}$. The inner cortical properties were assumed to be equivalent to that of the outer cortical due their negligible difference. The stiffest direction was assumed to be radial, with the transverse stiffness being the tangential direction. For pediatric skulls, due to the lack of available anisotropic data, the anisotropic ratios $E_{1} / E_{t}$ was taken from Peterson and applied to isotropic data gathered by Marguleis [40], Mcpherson [41], Peterson [39], Davis [24] and Chen [42] as shown in Figure 8(c). A monomolecular fit was used to model the relationship between the stiffest Young's modulus and age with a resulting R-squared value of 0.99

An anisotropic yield surface was assumed for cortical bone. Reilly [43] showed that compact bone collected from human femurs loaded in the longitudinal direction failed at a higher stress compared to being loaded in the transverse axis. Thus in order to describe the yield surface for cortical bone, a Hill's anisotropic yield criterion was utilized. Since the cortical bone exhibits tension-compression asymmetry in yielding as shown in Reilly [43], a generalized Hill's criterion is required to describe the yield surface. However, since the nature of the clamping procedure produces mostly compressive stresses within the material, this asymmetry was ignored. The Hill's parameters[44] used are presented in Table 1.

Table 1: Hill's parameters used in model

\begin{tabular}{|c|c|c|c|c|c|}
\hline F & G & H & L & M & N \\
\hline 1.95 & 0.5 & 0.5 & 3.67 & 1.5 & 1.5 \\
\hline
\end{tabular}

To account for the effect of age on the yield strength of bone, a survey was conducted on the available literature. It was found that from 0 to 6 years of age the ultimate strength of bone can be modelled as a linear relation with an R-squared value of 0.97. Due to the lack of available data for cortical parietal bones for 
adults. The ultimate strength after 6 years of age is assumed to be constant and equal to $185 \mathrm{MPa}$. The data surveyed is from Marguleis [40,45] and Davis [24] as shown in Figure 8(b). The ultimate strength was taken to be equal to the yield strength as it was shown in Reilly [43] to be a close approximation, from the stress strain curves.

The von-Mises yield criterion was assumed for the trabecular bone. Due to the lack of data on the evolution of apparent trabecular tissue properties with age, we assumed constant isotropic trabecular mechanical properties with age for the simulations with typical trabecular bone properties $(\mathrm{E}=450$ $\mathrm{MPa}, \sigma_{\mathrm{u}}=5 \mathrm{MPa}, \mathrm{v}=0.33$ ) [38].

The post yield behavior for both cortical and trabecular bone was assumed to be almost perfectly plastic. As it was shown in the stress-strain curves of cortical bone presented by Reilly [43]
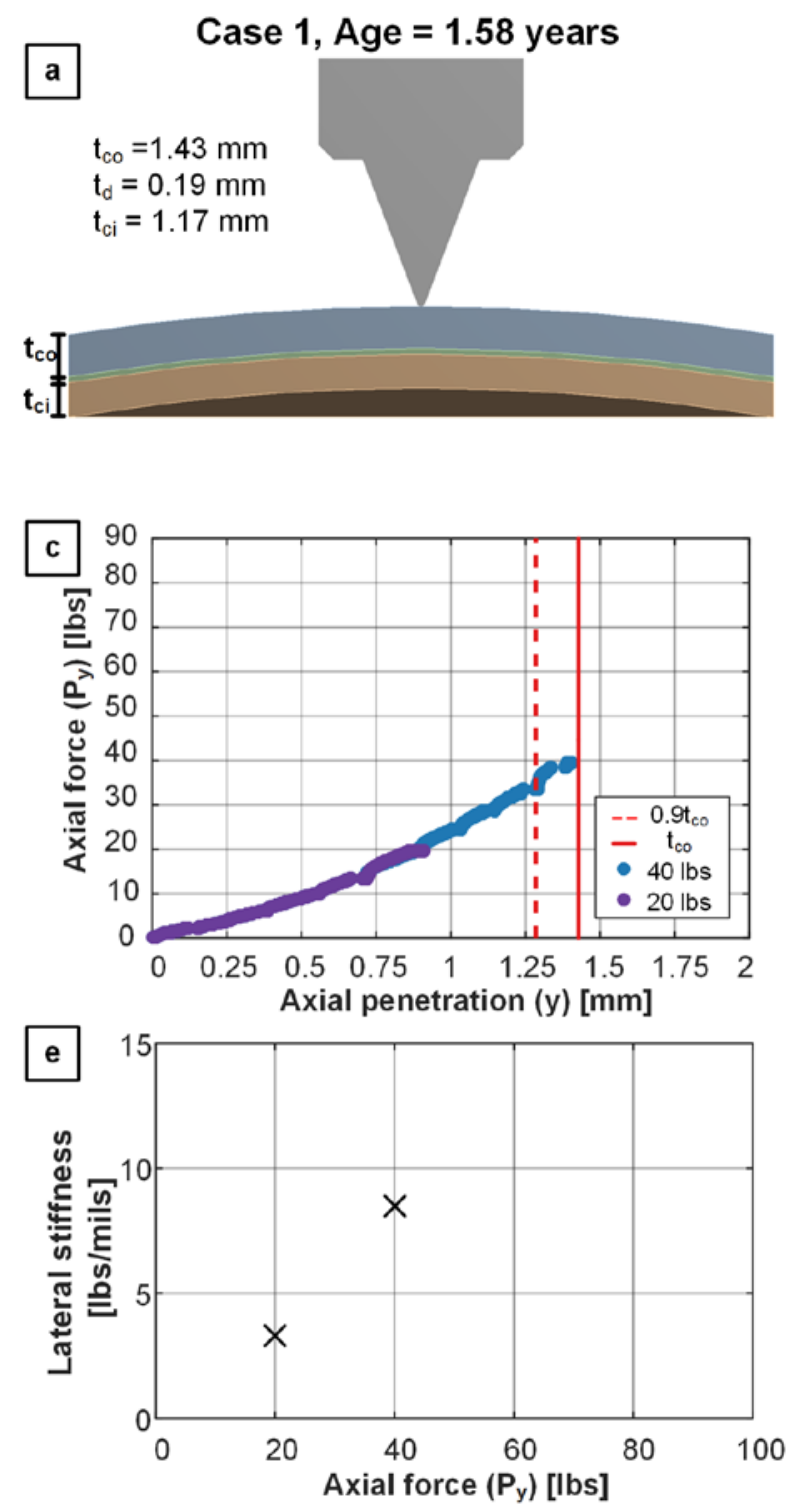

to be a fair approximation to the behavior of yielded bone under compression. A tangent modulus of $1 \mathrm{MPa}$ was assumed for the cortical bone which was multiple orders of magnitude smaller than the smallest Young's modulus used. While a tangent modulus of $10 \mathrm{MPa}$ was assumed for the trabecular bone.

The pin selected is a pediatric type titanium pin with isotropic properties $\left(\mathrm{E}=96 \mathrm{GPa}, \sigma_{\mathrm{y}}=930 \mathrm{MPa}, \mathrm{v}=0.36\right)$.

\section{RESULTS AND DISCUSSION}

To test the model, we ran two FEM simulations corresponding to 1.58 and 12.17 years of age. These specific ages were selected to correspond to actual ages in case studies encountered by the authors [12]. The schematic for the cases as well as the resulting axial force vs. penetration as well as well the lateral stiffness vs. applied axial force are shown in Figure 9.

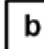

Case 2, Age $=12.17$ years
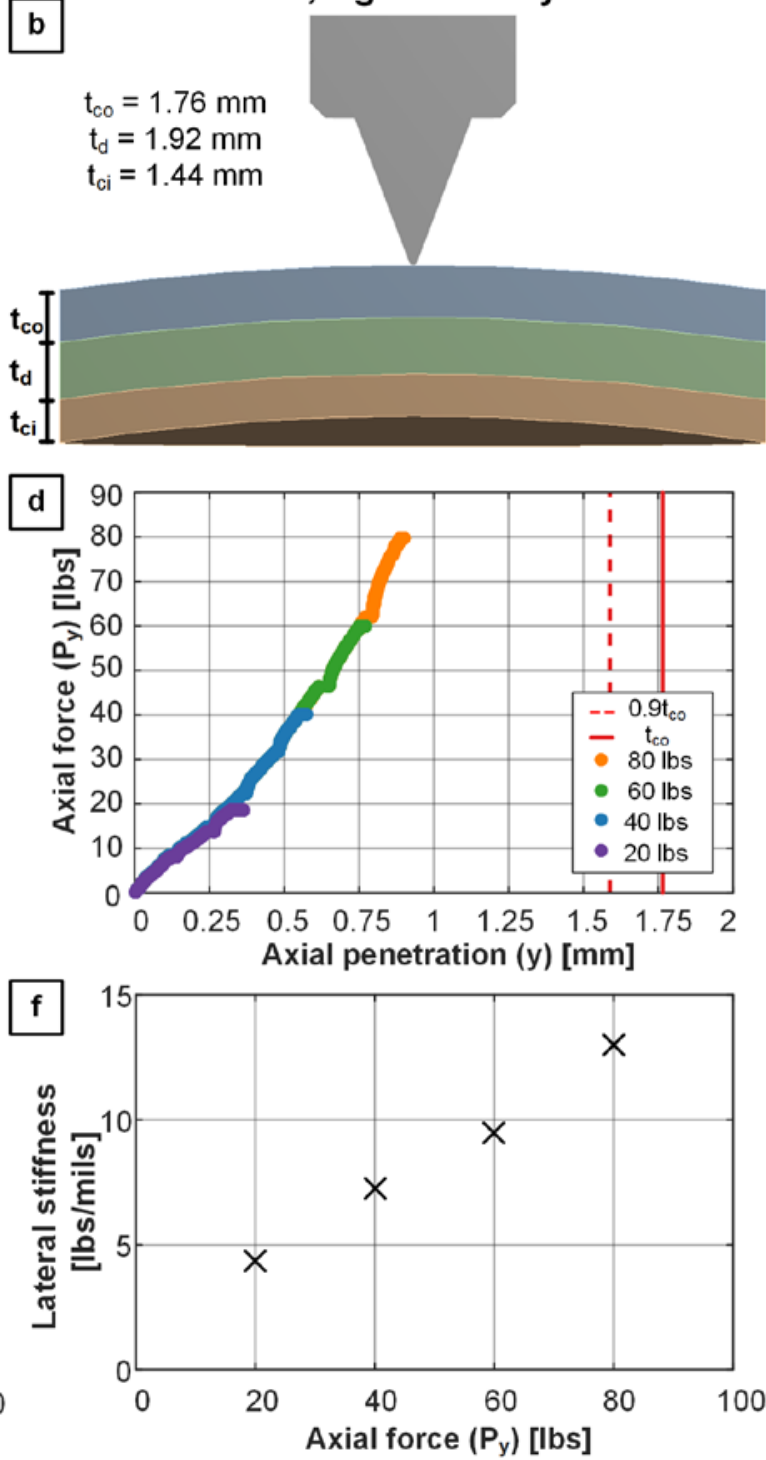

Figure 9: Simulation results: (a,b) Schematics for cases 1 and 2 respectively, (c,d) FEM results of of axial penetration response to different axial force input for case 1 and 2 respectively, (e,f) Lateral stiffness of bone at different axial forces 
From the force displacement curve for both cases (Figure 9(c,d)), the material is noted to stiffen with increased depth; requiring a larger amount of lateral loading to get the same displacement at later stages of loading. This could be attributed to the pile up of the material with lateral movement of the pin, and thus the compressed material pushes back on the pin. For both cases, penetration didn't exceed the outer cortical layer. The discontinuities observed in the solution are attributed to the remeshing of the domain, since equilibrium calculations after remeshing could lead to small pin jumps. For case 1 at $35 \mathrm{lbs}$ of load, pin penetration exceeded the safety threshold at $90 \%$ of outer cortical layer. In contrast for case 2, where even maximum loading, the penetration achieved did not exceed the safety threshold. This results indicates that generally, the same clamping forces would cause higher penetrations for young children, prompting extra caution when tightening the pins in pediatric patients.

It is also noted from the lateral stiffness curves that increased penetration and axial force brings a greater degree of resistance to lateral movement of the pin, implying more stable lateral fixation. This is explained in terms of increased penetration into the cortical layer, which brings about an increased contact area between the pin and bone. There is thus more material to push back against the pin once a lateral load is applied. It should be noted that the axial stiffness is not a sufficient measure for slip resistance. However, It is evident that at small axial loads like at an axial load of $20 \mathrm{lbs}$ in case 2, the penetration depth is very low $(y=375 \mu \mathrm{m})$ and the applied lateral force are within range of actual scratching experiments in literature [46]. This implies that at low penetrations, lateral forces would be sufficient to trigger slipping of the pins and patient injury. The von-Mises stress contours are shown at different stages of the simulation of age 21 in Figure 10, showing the evolution of yielded material with increased penetration.

\section{CONCLUSIONS}

In this work, we present a root cause analysis for pin type HIDs with emphasis on biomechanical risk factors and causes. To address the biomechanical factors, we developed a FEM model of a single pin penetrating a skull disc. The model incorporates age dependent geometrical and mechanical properties to investigate the effect of age on complication risk. The model outputs the change in axial penetration with applied axial force as well as the lateral stiffness of the bone under full head weight. While the model doesn't incorporate fracture mechanics to assess fracture risk, it provides insights by investigating the age dependence on excessive penetration and pin stability. Results show that age is a major factor in the clamping process, where pediatric patients have a higher risk of dural penetration than adult patients, due to softer bone structures. Pin stability under lateral load is also assessed through measuring the lateral stiffness under head weight. It is also evident that pin stability is higher at higher loads but at higher excessive penetration risk, indicating the need for a study to assess the trade-offs between the risk of excessive penetration and the risk of pin slip. Future work includes using the model to assess critical ages for pin use by running the model for a range of ages. The effect of pin shape on the penetration will also be investigated using our model. The effect of pin placement could be assessed using a full skull model incorporating three pins.

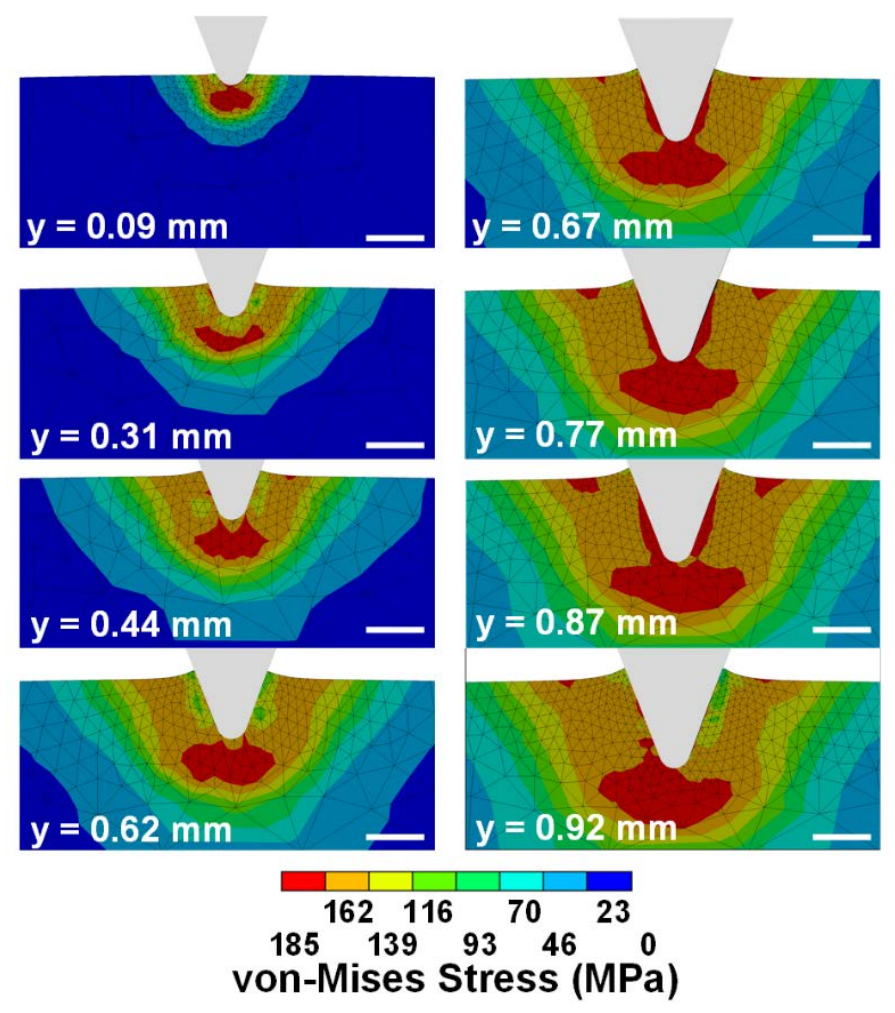

Figure 10: von-Mises stress of the outer cortical layer at different stages of simulation at maximum axial load $\left(P_{\mathbf{y}}\right)$ of 80 lbs for age 21 years. Length bar $(0.5 \mathrm{~mm})$

\section{ACKNOWLEDGMENTS}

This research was supported in part by the University of Pittsburgh Center for Research Computing through the resources provided.

\section{REFERENCES}

[1] Gupta, N., 2006, “A Modification of the Mayfield Horseshoe Headrest Allowing Pin Fixation and Cranial Immobilization in Infants and Young Children," Neurosurgery, 58(SUPPL. 1), pp. 1-4.

[2] Pang, D., 1982, "Air Embolism Associated with Wounds from a Pin-Type Head-Holder,” J. Neurosurg., 57(5), pp. 710-713.

[3] Lee, M., Rezai, A. R., and Chou, J., 1994, "Depressed Skull Fractures in Children Secondary to Skull Clamp Fixation Devices.," Pediatr. Neurosurg., 21(3), p. 174-7; discussion 178.

[4] Baerts, W. D. M., Lange, J. J. de, Booij, L. H. D. J., and Broere, G., 1984, "Complications of the Mayfield Skull Clamp,” Anesthesiology, 61(1), pp. 460-461.

[5] Berry, C., Sandberg, D. I., Hoh, D. J., Krieger, M. D., 
and McComb, J. G., 2008, "Use of Cranial Fixation Pins in Pediatric Neurosurgery.," Neurosurgery, 62(4), pp. 913-8-9.

[6] McClain, C. D., Soriano, S. G., Goumnerova, L. C., Black, P. M., and Rockoff, M. A., 2007, "Detection of Unanticipated Intracranial Hemorrhage during Intraoperative Magnetic Resonance Image-guided Neurosurgery,” J. Neurosurg. Pediatr., 106(5), pp. 398400.

[7] Anegawa, S., Shigemori, M., Yoshida, M., Kojo, N., Torigoe, R., Shirouzu, T., and Kuramoto, S., 1986, "[Postoperative Tension Pneumocephalus--Report of 3 Cases].,” No Shinkei Geka., 14(8), pp. 1017-22.

[8] Vitali, A. M., and Steinbok, P., 2008, “Depressed Skull Fracture and Epidural Hematoma from Head Fixation with Pins for Craniotomy in Children," Child's Nerv. Syst., 24(8), pp. 917-923.

[9] Yan, H. J., 2007, "Epidural Hematoma Following Use of a Three-Point Skull Clamp,” J. Clin. Neurosci., 14(7), pp. 691-693.

[10] Medina, M., Melcarne, A., Musso, C., and Ettorre, F., 1997, “Acute Brain Swelling during Removal of Supratentorial Cystic Lesion Caused by Contralateral Extradural Hematoma: Case Report,” Surg. Neurol., 47(5), pp. 428-431.

[11] Martínez-Lage, J. F., Almagro, M. J., Serrano, C., and Mena, L., 2011, "Depressed Skull Fracture by a ThreePin Head Holder: A Case Illustration,” Child's Nerv. Syst., 27(1), pp. 163-165.

[12] Zaazoue, M. A., Bedewy, M., and Goumnerova, L. C., 2017, "Complications of Head Immobilization Devices in Children: Contact Mechanics, and Analysis of a Single Institutional Experience,” Neurosurgery, 0(0), pp. $1-8$.

[13] U.S. Food \& Drug Adminstration, 2016, "Safety Communications - Neurosurgical Head Holders (Skull Clamps) and Device Slippage: FDA Safety Communication” [Online]. Available: http://www.fda.gov/MedicalDevices/Safety/AlertsandN otices/ucm487665.htm. [Accessed: 10-Feb-2017].

[14] McFadden, J. T., 2001, "SURGICAL HEAD CLAMPING DEVICE.”

[15] Sklar, F., Dinkler, I. C. E., and Easton, K. R., 2005, "Head Support and Stabilization System."

[16] Solomon, C. T., and Solomon, T. C., 2012, "SKULL CLAMP SYSTEM WITH PRESSURE LIMITING AND ALARM SYSTEMS.”

[17] Sgouros, S., Grainger, M. C., and McCallin, S., 2005, "Adaptation of Skull Clamp for Use in Image-Guided Surgery of Children in the First 2 Years of Life,” Child's Nerv. Syst., 21(2), pp. 148-149.

[18] Ebraheim, N. A., Liu, J., Patil, V., Hiltner, L., Shapiro, A., Zamary, K. R., Dick, D. C., and Yeasting, R. A., 2009, “An Evaluation of Halo Pin Insertion Torque on Outer Table Penetration in Elderly Patients,” J. Spinal Disord. Tech., 22(3), pp. 177-181.
[19] Cranial Stabilization Product Catalogue, Integra LifeSciences Services.

[20] Beall, M. S., Copley, L. A. B., Niemann, J. J., Lankachandra, K., and Williams, J. L., 2007, "Biomechanical Evaluation of Fetal Calf Skull as a Model for Testing Halo-Pin Designs for Use in Children,” J. Biomech., 40(5), pp. 1137-1144.

[21] Skull Clamp REF ( A3059) Instruction Manual, Integra LifeSciences Services.

[22] Voor, M. J., Anderson, R. C., and Hart, R. T., 1997, "Stress Analysis of Halo Pin Insertion by Non-Linear Finite Element Modeling,” J. Biomech., 30(9), pp. 903909.

[23] Loder, R. T., 1996, "Skull Thickness and Halo-Pin Placement in Children: The Effects of Race, Gender, and Laterality,” J. Pediatr. Orthop., 16(3), pp. 340-343.

[24] Davis, M. T., Loyd, A. M., Shen, H. H., Mulroy, M. H., Nightingale, R. W., Myers, B. S., and Bass, C. D., 2012, "The Mechanical and Morphological Properties of 6 Year-Old Cranial Bone,” J. Biomech., 45(15), pp. 24932498.

[25] Li, Z., Park, B. K., Liu, W., Zhang, J., Reed, M. P., Rupp, J. D., Hoff, C. N., and Hu, J., 2015, “A Statistical Skull Geometry Model for Children 0-3 Years Old,” PLoS One, 10(5), pp. 1-13.

[26] Garfin, S. R., Roux, R., Botte, M. J., Centeno, R., and Woo, S. L., 1986, "Skull Osteology as It Affects Halo Pin Placement in Children.,” J. Pediatr. Orthop., 6(4), pp. 434-6.

[27] Wong, W. B., and Haynes, R. J., 1994, “Osteology of the Pediatric Skull. Considerations of Halo Pin Placement," Spine (Phila Pa 1976), 19(13), pp. 1451-1454.

[28] De Souza Fernandes, A. C., Neto, A. I. T., De Freitas, A. C., and De Moraes, M., 2011, "Dimensional Analysis of the Parietal Bone in Areas of Surgical Interest and Relationship between Parietal Thickness and Cephalic Index,” J. Oral Maxillofac. Surg., 69(11), pp. 29302935.

[29] Moreira-Gonzalez, A., Papay, F. E., and Zins, J. E., 2006, "Calvarial Thickness and Its Relation to Cranial Bone Harvest,” Plast. Reconstr. Surg., 117(6), pp. 1964-1971.

[30] Sullivan, W. G., and Smith, A. A., 1989, “The Split Calvarial Graft Donor Site in the Elderly: A Study in Cadavers.,” Plast. Reconstr. Surg., 84(1), pp. 29-31.

[31] Voie, A., Dirnbacher, M., Fisher, D., and Hölscher, T., 2014, "Parametric Mapping and Quantitative Analysis of the Human Calvarium,” Comput. Med. Imaging Graph., 38(8), pp. 675-682.

[32] Lillie, E. M., Urban, J. E., Lynch, S. K., Weaver, A. A., and Stitzel, J. D., 2016, "Evaluation of Skull Cortical Thickness Changes with Age and Sex from Computed Tomography Scans,” J. Bone Miner. Res., 31(2), pp. 299-307.

[33] Lee, A. H., 2007, “Interplay between Growth and Mechanics in the Evolution of Bone Microstructure in Dinosaurs,” p. 210. 
[34] Cooper, L. N., Lee, A. H., Taper, M. L., and Horner, J. R., 2008, "Relative Growth Rates of Predator and Prey Dinosaurs Reflect Effects of Predation,” Proc. R. Soc. B Biol. Sci., 275(1651), pp. 2609-2615.

[35] Erickson, G. M., 2014, “On Dinosaur Growth,” Annu. Rev. Earth Planet. Sci., 42(1), pp. 675-697.

[36] Høye, T. T., and Forchhammer, M. C., 2006, "Early Developed Section of the Jaw as an Index of Prenatal Growth Conditions in Adult Roe Deer Capreolus Capreolus,” Wildlife Biol., 12(1), pp. 71-76.

[37] Huang, A. H., Sun, H. H., Skolnick, G. B., and Woo, A. S., 2015, "Thickness of Calvarium and Diploic Space in Children Ages 0 to 17 as Assessed by Computed Tomography,” Eur. J. Plast. Surg., 38(3), pp. 193-198.

[38] Cowin, S. C., ed., 2001, Bone Mechanics Handbook, Taylor \& Francis.

[39] Peterson, J., and Dechow, P. C., 2002, "Material Properties of the Inner and Outer Cortical Tables of the Human Parietal Bone,” Anat. Rec., 268(1), pp. 7-15.

[40] Margulies, S. S., and Thibault, K. L., 2000, "Infant Skull and Suture Properties: Measurements and Implications for Mechanisms of Pediatric Brain Injury,” J. Biomech.
Eng., 122(4), p. 364.

[41] McPherson, G. K., and Kriewall, T. J., 1980, “The Elastic Modulus of Fetal Cranial Bone: A First Step towards an Understanding of the Biomechanics of Fetal Head Molding,” J. Biomech., 13(1), pp. 9-16.

[42] Chen, Y., 2014, "Mechanical Properties of Cranial Bones and Sutures in 1-2-Year-Old Infants,” Med. Sci. Monit., 20, pp. 1808-1813.

[43] Reilly, D. T., and Burstein, A. H., 1975, "The Elastic and Ultimate Properties of Compact Bone Tissue,” J. Biomech., 8(6), pp. 393-405.

[44] Hill, R., 1948, "A Theory of the Yielding and Plastic Flow of Anisotropic Metals,” Proc. R. Soc. A Math. Phys. Eng. Sci., 193(1033), pp. 281-297.

[45] Coats, B., and Margulies, S. S., 2006, "Material Properties of Human Infant Skull and Suture at High Rates,” J. Neurotrauma, 23(8), pp. 1222-1232.

[46] Kataruka, A., Mendu, K., Okeoghene, O., Puthuvelil, J., and Akono, A. T., 2017, "Microscopic Assessment of Bone Toughness Using Scratch Tests,” Bone Reports, 6, pp. 17-25. 